\title{
EPOC: Caminata de 6 minutos y su relación con mortalidad en pacientes con enfermedad pulmonar obstructiva crónica (EPOC)
}

\section{Six minute walk test and its relation to mortality in chronic obstructive pulmonary disease patients}

Lisseth Fernanda Marin Carrillo, MD.(1); Mary Bermúdez Gómez, MD.(2)

Rev Colomb Neumol 2013; 24 (4):198-199

\section{REFERENCIA}

Polkey MI, Spruit MA, Edwards LD, Watkins ML, Pinto-Plata V, Vestbo J, Calverley PM, Tal-Singer R, Agusti A, Bakke PS, Coxson HO, Lomas DA, MacNee W, Rennard S, Silverman EK, Miller BE, Crim C, Yates J, Wouters EF, Celli B. Evaluation of COPD Longitudinally to Identify Predictive Surrogate Endpoints (ECLIPSE) Study Investigators. Six minute walk test in COPD: minimal clinically important difference for death or hospitalization. Am J Respir Crit Care Med. 2013; 187: 382-6.

\section{PREGUNTA}

¿Cuál es el cambio mínimo clínicamente significativo en la caminata de 6 minutos, que se correlaciona con mayor riesgo de hospitalización y/o mortalidad en pacientes con EPOC?

\section{DISEÑO}

Estudio analítico multicéntrico, longitudinal, a partir de la cohorte prospectiva ECLIPSE (de la sigla del inglés Evaluation of COPD Longitudinally to Identify Predictive Surrogate Endpoints).

\section{PACIENTES}

Se incluyeron 1.847 pacientes que corresponden a una subpoblación de la cohorte del estudio ECLIPSE, con edad entre 40 y 75 años, historia de tabaquismo de $\geq 10$ paquete año, $V E F_{1}<80 \%$ y relación $V E F_{1}$ I CVF $\leq 0,7$.

\section{INTERVENCIÓN}

Realización de la prueba de caminata de 6 minutos (C6M) basal y luego cada año. Determinación basal, a los 3 y 6 meses y luego semestralmente del VEF1 en valores absolutos y porcentaje mediante espirometría y medición de calidad de vida utilizando el cuestionario de Saint George.

\section{DESENLACES}

Mortalidad y frecuencia de hospitalización durante el tiempo de seguimiento.

\section{SEGUIMIENTO}

Tres años.

\section{CONCLUSIÓN}

La disminución de 30 metros o más en la C6M registrada en el año inmediatamente anterior, se relaciona con riesgo de mortalidad (HR 1,93; IC95\%1,29-2,9).

No se encontró aumento del riesgo de hospitalización por exacerbación en relación con el cambio mínimo clinicamente significativo, y la asociación entre

\footnotetext{
(1) Residente de segundo año de Medicina Interna, Pontificia Universidad Javeriana, Hospital Universitario San Ignacio, Bogotá, Colombia.

(2) Profesor Titular Medicina Interna y Neumologia, Decana Académica Facultad de Medicina, Pontificia Universidad Javeriana, Hospital Universitario San Ignacio, Bogotá, Colombia.
} 
Tabla. Cambio mínimo significativo de -30 metros y su relación con los desenlaces.

\begin{tabular}{|lccc|}
\hline \multicolumn{1}{c}{ Desenlace } & HR & IC $\mathbf{~ 9 5 \%}$ & Valor de $\boldsymbol{p}$ \\
\hline Mortalidad & 1,93 & $1,29-2,90$ & 0,001 \\
Hospitalización por exacerbación & 1,18 & $0,93-1,49$ & 0,179 \\
Mortalidad y hospitalización por exacerbación & 1,24 & $0,99-1,54$ & 0,056 \\
\hline
\end{tabular}

la caminata de 6 minutos, el VEF, y el cuestionario de Saint George fue débil. Los valores de HR para los desenlaces medidos en relación con el cambio mínimo significativo (disminución de $30 \mathrm{~m}$ ) se ilustran en la tabla.

\section{COMENTARIO}

La caminata de 6 minutos (C6M) es una prueba de ejercicio submáximo que refleja la capacidad de ejercicio de los pacientes con enfermedades respiratorias, incluyendo la EPOC, en donde muestra correlación con la severidad del compromiso pulmonar y extrapulmonar. Es útil también en el seguimiento de pacientes con fibrosis pulmonar e hipertensión pulmonar, para establecer el riesgo de mortalidad. La distancia caminada durante la prueba, predice deterioro clínico y mortalidad y, así mismo, permite evaluar el impacto de intervenciones terapéuticas (1).

El cambio mínimo clínicamente significativo es un parámetro útil que se obtiene durante el seguimiento de los pacientes. Ha sido reportado en estudios previos al análisis de la cohorte ECLIPSE, con poblaciones selectas de pacientes de pequeñas cohortes en escenarios como rehabilitación pulmonar y cirugia de reducción de volumen pulmonar. Su valor fue establecido por Redelmeier y colaboradores en 54 metros, con un rango entre 30 y 72 metros $(2,3)$.

Lo innovador del estudio ECLIPSE, es que antes de su análisis no se había intentado establecer la relación entre el cambio minimo clinicamente significativo y los desenlaces de mortalidad y hospitalización por exacerbación. Los reportes previos hacian relación al valor de la caminata de 6 minutos con otros índices de función pulmonar como medida de efectividad de intervenciones, sin grupo control. Otros autores han reportado su correlación con parámetros espirométricos en pacientes con EPOC severa y muy severa (4).

Para el análisis del estudio sólo se tuvieron en cuenta las exacerbaciones que requirieron hospitali- zación, partiendo del hecho que la obtención de datos se realizó de forma retrospectiva y la relevancia otorgada por el paciente. No hay reporte de los eventos que requirieron manejo antibiótico o con corticoide, lo que pudo haber influido en la pobre relación entre el cambio mínimo significativo de importancia clínica y el riesgo de exacerbación. Para los autores, la ausencia de relación pudo deberse a diferentes impactos de la terapia y a los distintos criterios de hospitalización en los centros participantes del estudio ECLIPSE.

El desenlace de mortalidad registró mortalidad total independiente de la causa, y no se discriminó de acuerdo con la etiología de la muerte. Sin embargo y de acuerdo con los autores, el valor agregado de la C6M y su asociación con mortalidad, parte de la capacidad de esta prueba para reflejar el grado de compromiso pulmonar y extrapulmonar por enfermedad y comorbilidades.

La C6M y el cambio mínimo clínicamente significativo, como indice de severidad de compromiso pulmonar y predictor de mortalidad, es suficiente razón para impulsar su uso como herramienta de evaluación en los pacientes con EPOC.

\section{BIBLIOGRAFIA}

1. Golpe R, Pérez-de-Llano LA, Méndez-Marote L, Veres-Racamonde A. Prognostic significance of distance, work, oxygen saturation and dyspnea during 6 minute walk test in COPD patients. Respir Care. 2013 Jan 15. [Epub ahead of print]

2. Redelmeier DA, Bayoumi AM, Goldstein RS, Guyatt GH. Interpreting small differences in functional status: the six minute walk test in chronic lung disease patients. Am J Respir Crit Care Med 1997: 155: 1278-82.

3. Lisboa C, Barria P, Yáñez J, Aguirre M, Díaz O. La prueba de caminata en seis minutos en la evaluación de la capacidad de ejercicio en pacientes con enfermedad pulmonar obstructiva crónica. Rev Méd Chile. 2008; 136: 1056-1064.

4. Chen H, Liang BM, Tang YJ, Xu ZB, Wang K, Yi Q, Ou XM, Feng $\mathrm{YL}$. Relationship between 6-minute walk test and pulmonary function test in stable chronic obstructive pulmonary disease with different severities. Chin Med J (Engl). 2012; 125: 3053-8. 